\title{
EFFECTS OF TRABECULAR BONE STRUCTURE ON ITS FATIGUE LIFE IN TEST WITH STEPWISE INCREASING AMPLITUDE
}

\author{
T. Małek ${ }^{*}$, M. Slomion ${ }^{* *}$, A. Mazurkiewicz ${ }^{* * *}$
}

\begin{abstract}
The study presents the evaluation of the relationship between the fatigue life of the trabecular bone determined in a compression test with a stepwise increasing amplitude and the indices defining the structure of bovine trabecular bone. The study included 32 samples collected from femoral head. The tests using a micro-CT scanner were carried out to determine the structural parameters defining the internal structure of the trabecular bone samples. The following parameters were evaluated: bone volume ratio $B V / T V$, trabecular thickness Tb.Th, trabecabecular number Tb.N and separation Tb.Sp. The samples were subjected to a fatigue test under load with a stepwise increasing amplitude. The fatigue life was determined for each sample. The relationships between the parameters defining the internal structure of the bone and its fatigue life were determined using a determination coefficient $R^{2}$ and was in range $R^{2}=0.61-0.69$.
\end{abstract}

Keywords: trabecular bone, structure parameters, fatigue test, stepwise increasing amplitude

\section{Introduction}

A trabecular bone is a porous structure found inside human or animal bones in the epiphyses of long bones and inside the short, flat and irregular bones. Its internal structure depends on many factors, including age, past diseases, lifestyle and activity levels. It is constantly rebuilt throughout the human life span. It is estimated that approximately $25 \%$ of its volume is rebuilt in a year (Covin, 1999). Any changes in its structure and volume will affect the overall strength of the entire bone. Thus, knowing the relationship between its structure and its strength properties is essential (Goulet et al., 1994 and Covin, 1999). The purpose of the study was to determine the relationship between the internal structure of the bone and its fatigue life. Knowledge of these relationships will allow to understand the relationship between the strength properties of the trabecular bone and its structure.

\section{Methods}

The study included 32 samples of bovine trabecular bone. The samples were collected from femoral heads within 24 hours from slaughter. All animals were from the same herd, were at a similar age and of the weight. The first was cut slice perpendicularly to the axis of bone neck. Next from the slice were cut samples. The samples were cylindrical in shape with $10 \mathrm{~mm}$ diameter and height. Figure 1 shows the sample collecting method. The collected samples were frozen at $-20{ }^{\circ} \mathrm{C}$.

MSc Tomasz Małek, PhD candidate: Member of Scientific Group BioMed, University of Sciences and Technology, Kaliskiego 7 Street; 85-796, Bydgoszcz; PL, e-mail: tomasz.m.malek@gmail.com

** MSc Malgorzata Slomion, PhD candidate: Member of Scientific Group BioMed, University of Sciences and Technology, Kaliskiego 7 Street; 85-789 Bydgoszcz; PL, e-mail: m.slomion@yahoo.pl

*** Assoc. Prof. Adam Mazurkiewicz, PhD: Department of Mechanical Engineering, University of Sciences and Technology, Kaliskiego 7 Street; 85-789 Bydgoszcz; PL, e-mail: adam.mazurkiewicz@utp.edu.pl 


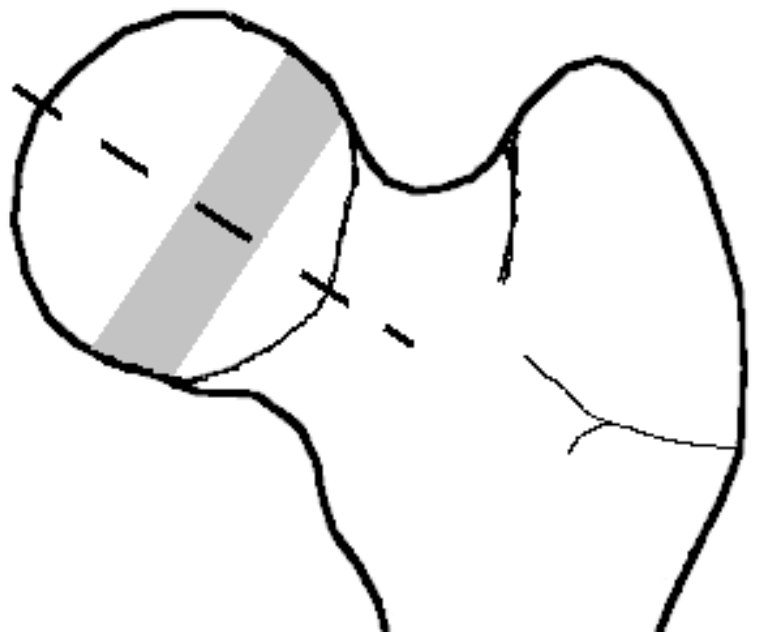

Fig. 1: Samples collecting scheme.

The structure parameters were measured using Skyscan 1172 micro-CT scanner (Brucker, Belgium). The samples were scanned with $20 \mu \mathrm{m}$ resolution. The following parameters were determined for each sample (Cichański et al., 2010 and Goulet et al., 1994 and Lee, 2013).

- Trabecular number - average number of trabeculae in structure, $1 / \mathrm{mm}$;

- Trabecular thickness - average thickness of trabeculae in structure, mm;

- Trabecular separation - average separation between trabeculae in structure;

- Bone volume ratio - bone tissue volume / trabeculae (sample) volume, -.

Each sample was subjected to a compression test with a gradually increasing amplitude. The test program was carried out as follows: an initial $5 \mathrm{~N}$ force was applied to the sample and the strain of the sample at the initial force was taken as a "zero" point. The compressive load was divided into the blocks of 500 cycles. In the first block, the compressive load was max. $5 \mathrm{~N}$ and min. $0 \mathrm{~N}$, whereas in each subsequent block, the maximum load was increased by $10 \mathrm{~N}$. The blocks were completed in turn. An increase in strain in the 250th loop was recorded for each block. The increase was linear for the first several blocks and a median was calculated for the increase. The loop during which an increase in strain has exceeded the estimated linear increase by 10\% was taken as a fatigue life criterion (Topoliński et al., 2011 and 2012). The test was carried out at $1 \mathrm{~Hz}$ frequency using Instron E3000 testing system (Instron, High Wycombe, England).

The results were included in the Shapiro-Wilk test at $\mathrm{P}=0.05$ to determine if the distribution of the results is normal.

\section{Results}

Table 1 shows the values structure parameters obtained using micro-CT tests and value of fatigue life Ns. The average, minimum and maximum, standard deviation were calculated.

Table 1:Values of structure parameters of trabecular bone.

\begin{tabular}{lccccc}
\hline & BV/TV, - & Tb.Th, mm & Tb.N, 1/mm & Tb.Sp, mm & Ns, cycle \\
\hline Average (SD) & $0.203(0.044)$ & $0.233(0.058)$ & $0.845(0.220)$ & $0.938(0.236)$ & 37351 \\
& & & & & $(16832)$ \\
\hline Min / Max & $0.136 / 0.325$ & $0.124 / 0.311$ & $0.554 / 1.341$ & $0.588 / 1.23$ & $14567 /$ \\
& & & & & 78453 \\
\hline
\end{tabular}

SD - standard deviation 
The test results Shapiro-Wilk test showed a normal or log-normal distribution of the results for each structure index and fatigue life. Therefore, relationships between the indices od structure and the fatigue life was determined using a determination coefficient $\mathrm{R}^{2}$.

Table 2 shows the relationships between the determined structure parameters and the fatigue life. The best relationship was determined between the fatigue life and bone volume ratio BV/TV. The determination coefficient $\mathrm{R}^{2}$ of the relationship was 0.69 .

Table 2:Values $R^{2}$ coefficient between structure parameters and fatigue life.

\begin{tabular}{lcccc}
\hline & BV/TV & Tb.Th & Tb.N & Tb.Sp \\
\hline Fatigue life, Ns & 0.69 & 0.62 & 0.65 & 0.61 \\
\hline
\end{tabular}

Figures 2 and 3 shows the example relationship between the determined fatigue life and trabecular number Tb.N and bone volume fraction BV/TV.

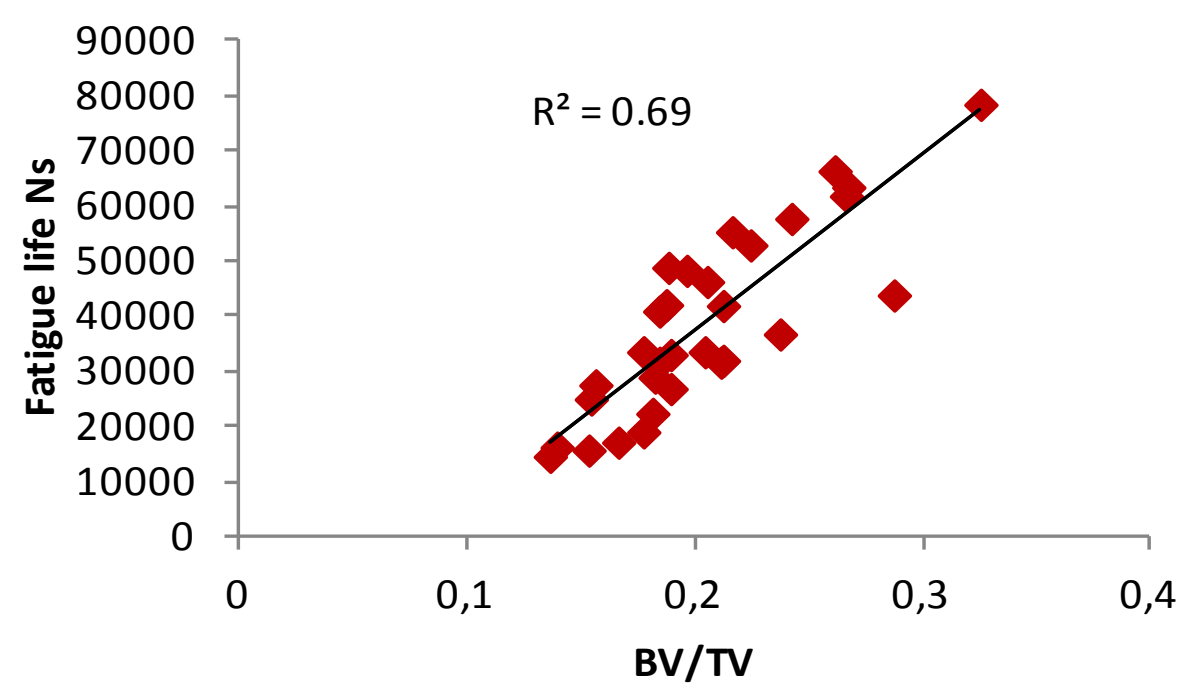

Fig. 2: Relationship between fatigue life and bone volume fraction BV/TV

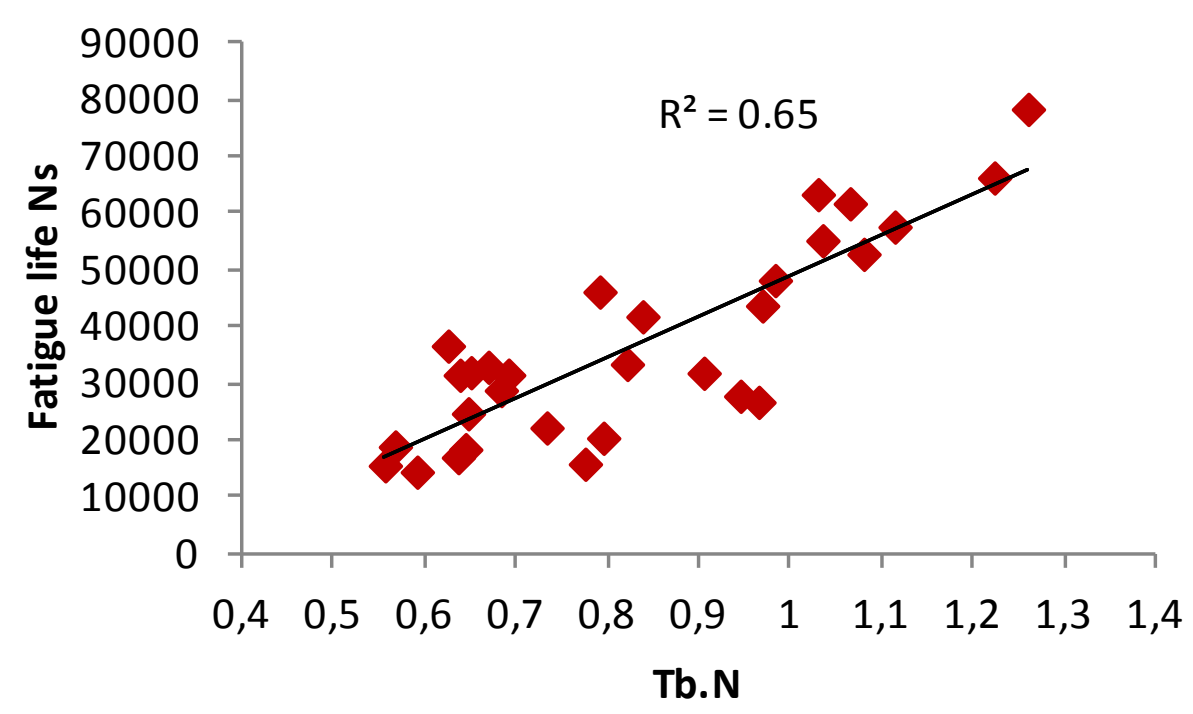

Fig. 3: Relationship between fatigue life and trabecular number Tb.N 


\section{Conclusions}

The results show that there is a relationship between the fatigue life of the trabecular bone determined in a cyclic compression test with a gradually increasing amplitude and the indices defining the structure of the trabecular bone. The best relationship was observed for the bone volume ratio. Standard deviation for each index was quite high which could be expected for the trabecular bone samples due to their individual traits (Covin, 1999 and An and Draugh, 1999).

The trabecular number, thickness and separation determines structure of trabeculae in the sample. Thus, it can be assumed that a structure with a lower number of larger structural elements will be more durable than a structure with a higher number of smaller elements. It is worth noting that the samples were taken and tested along the direction of the largest loads applied on the bone. In accordance with the Wolff's law, the bone strength in the direction of the main loads is the highest, since it corresponds to the trabecular trajectories. However, it affects other directions, and may weaken the entire bone. A more detailed evaluation of these relationships would require additional tests on a significantly larger number of samples collected in different directions in relation to the bone neck axis.

A bone volume ratio is a reciprocal of the porosity and determines the volume of the bone tissue in the sample volume. It follows a general trend whereby the higher the tissue volume in the bone volume the higher the strength.

In the compression test with a gradually increasing amplitude it is difficult to define the fatigue life criterion. Based on the previous studies, the authors have taken the loop during which an increase in strain has exceeded the estimated linear increase by $10 \%$ as a fatigue life criterion. This behaviour indicates a loss of load capacity of the structure in question. The values of the relationships between the indices defining the structure of the trabecular bone and its fatigue life determined in the test with a gradually increasing amplitude are similar to the values obtained by other researchers in the tests with a constant amplitude (An and Draugh, 1999). Thus, it can be assumed that the proposed fatigue life criterion is correct and can be used when testing the trabecular bone samples.

\section{References}

An, H. and Draugh, R. (1999) Mechanical testing of bone and the bone-implant interface. CRC Press, New York.

Cichanski, A., Nowicki K., Mazurkiewicz, A. And Topoliński T. (2010) Investigation of statistical relationships between quantities describing bone architecture, its fractal dimensions and mechanical properties. Acta Of Bioengineering And Biomechanics, 12, 4, pp. 69-77.

Covin, S. (1999) Bone mechanics handbook - second edition. CRC Press, New York.

Goulet, R. W., Goldstein, S. A., Ciarelli, M.J., Kuhn, J.L. (1994) The relationship between the structural and orthogonal compressive properties of trabecular bone. Journal of Biomechanics, 27, pp. 375-389.

Lee, K.I. (2013) Dependences of the backscatter coefficient on the frequency, the bone volume fraction, and the trabecular thickness in bovine femoral trabecular bone in vitro. Journal of the Korean Physical Society, 63, 5, pp. 991-995.

Topolinski, T., Cichanski, A., Mazurkiewicz, A. and Nowicki K. (2011) Fatigue Energy Dissipation in Trabecular Bone Samples with Step-wise-Increasing Amplitude Loading. Materials Testing, 53, 6, pp. 344-350.

Topolinski, T., Cichanski, A., Mazurkiewicz, A. et al. (2012) Applying a stepwise load for calculation of the S-N curve for trabecular bone based on the linear hypothesis for fatigue damage accumulation. Mater. Sci. Forum 726, pp. 39-42, doi:10.4028/www.scientific.net/MSF.726.39. 